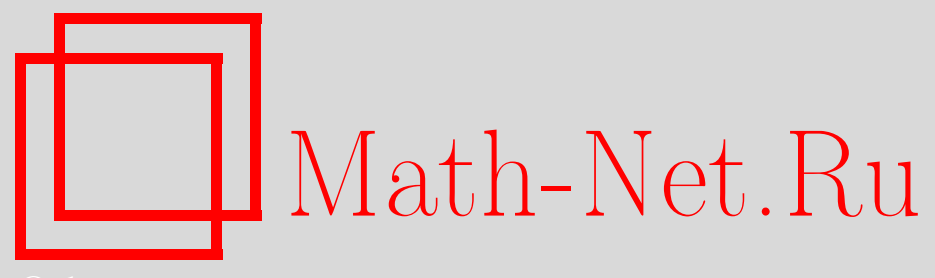

Ф. М. Малышев, Сложность восстановления начальных знаков фильтрующих генераторов одного класса, Матем. вопр. криптогр., 2015, том 6, выпуск 1, 109-116

DOI: https://doi.org/10.4213/mvk153

Использование Общероссийского математического портала Math-Net.Ru подразумевает, что вы прочитали и согласны с пользовательским соглашением http: //www . mathnet.ru/rus/agreement

Параметры загрузки:

IP : 44.207 .124 .84

26 апреля 2023 г., 17:06:43

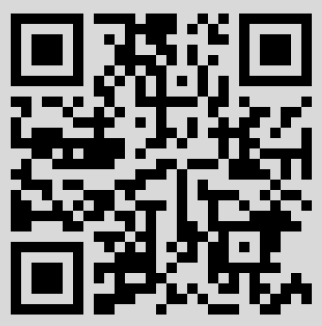




\title{
Сложность восстановления начальных знаков фильтрующих генераторов одного класса
}

\author{
Ф. М. Малышев \\ Математический институт им. В. А. Стеклова РАН, Москва
}

Получено 22.IV.2014

Решается задача восстановления начальных знаков рекуррентной последовательности порядка $m$ по значениям усложняющей выходной функции $F$. При естественном предположении о функции рекуррентной зависимости $f$ и функции $F$ эта задача решается с линейной по $m$ трудоемкостью, при этом требуется линейное же по $m$ число известных выходных знаков. Коэффициенты при $m$ однозначно задаются мощностями алфавита выходных знаков, алфавита знаков рекуррентной последовательности и числами аргументов в функциях $f$ и $F$.

Ключевые слова: рекуррентная последовательность, фильтрующий генератор, псевдослучайная последовательность

\section{The complexity of initial state recovery for a class of filter generators}

\section{F. M. Malyshev}

Steklov Mathematical Institute of RAS, Moscow

\begin{abstract}
A recovery problem for the initial state of the $m$-th order recurrent sequence from the output values of filter function $F$. Under natural conditions on the feedback function $f$ and filter function $F$ the complexity of initial state recovery from linear (in $m$ ) number of output values is shown to be linear in $m$. Coefficients of these linear functions are defined by the cardinalities of alphabet of output values, alphabet of input sequence elements and numbers of essential arguments of functions $f$ and $F$.
\end{abstract}

Key words: recurrent sequence, filter generator, pseudo-random sequence

Citation: Mathematical Aspects of Cryptography, 2015, vol. 6, no. 1, pp. 109-116 (Russian) 
В работе предлагается подход к решению задачи восстановления начальных знаков рекуррентных последовательностей порядка $m$ по выходам с усложняющих функций. В условиях, когда числа аргументов у усложняющей функции и у функции рекуррентной зависимости заданы, а сами эти функции удовлетворяют естественному предположению, получены линейные по $m$ верхние оценки трудоемкости и числа выходных знаков, необходимых для решения задачи.

Пусть $X$ и $Y$ - конечные алфавиты. Далее рассматриваются генераторы последовательностей $\left\{y_{i} \in Y, i \in \mathbb{Z}\right\}$, вычисляемых по формуле

$$
y_{i}=F\left(x_{i+l_{1}}, \ldots, x_{i+l_{r}}\right) \text {, }
$$

где $\left\{x_{i} \in X, i \in \mathbb{Z}\right\}$ - рекуррентная последовательность порядка $m$, удовлетворяющая рекуррентному соотношению

$$
x_{i+m}=f\left(x_{i}, x_{i+k_{1}}, \ldots, x_{i+k_{s}}\right), i \in \mathbb{Z},
$$

a $f: X^{s+1} \rightarrow X$ и $F: X^{r} \rightarrow Y$ - некоторые отображения. Обычно отображение $f\left(z_{1}, \ldots, z_{s+1}\right)$ является подстановочным по первой переменной $z_{1}$ при любой фиксации остальных $s$ переменных. Тогда последовательность $\left\{x_{i}, i \in \mathbb{Z}\right\}$ является рекуррентной и для обратного порядка элементов:

$$
x_{i}=f^{-}\left(x_{i+k_{1}}, \ldots, x_{i+k_{s}}, x_{i+m}\right), i \in \mathbb{Z}, f^{-}: X^{s+1} \rightarrow X .
$$

Номера $l_{1}, \ldots, l_{r}$ в задании функции съема $F$ (см. (1)) и номера $k_{1}, \ldots, k_{s}$ в задании рекуррентного соотношения (2) считаем упорядоченными:

$$
\begin{gathered}
0 \leq l_{1}<l_{2}<\ldots<l_{r} \leq m, \\
0<k_{1}<k_{2}<\ldots<k_{s}<m .
\end{gathered}
$$

Других условий на эти номера не накладывается.

Различные частные случаи таких генераторов псевдослучайных последовательностей представлены в монографии [1], где они называются фильтрующими генераторами.

Полученные в настоящей работе результаты основаны на соображениях работы [2] и относятся к ситуации, когда параметры $r \geq 1$ и $s \geq 1$ являются фиксированными, а порядок рекуррентной последовательности $m$ может принимать любые целочисленные значения.

Требования на отображения $f$ и $F$ не столь явные. Для их формулировки потребуются множества

$$
\Delta_{t}^{(s)}=\left\{\left(z_{1}, \ldots, z_{s}\right) \in \mathbb{Z}^{s} \mid z_{1} \geq 0, \ldots, z_{s} \geq 0, z_{1}+\ldots+z_{s} \leq t\right\} \subset \mathbb{Z}^{s},
$$




$$
\begin{gathered}
\Delta_{t}^{(s+1)}=\left\{\left(z_{0}, z_{1}, \ldots, z_{s}\right) \in \mathbb{Z}^{s+1} \mid z_{0} \geq 0, z_{1} \geq 0, \ldots, z_{s} \geq 0, z_{0}+z_{1}+\ldots+z_{s} \leq t\right\} \subset \mathbb{Z}^{s+1}, \\
t \in \mathbb{N} \cup\{0\} .
\end{gathered}
$$

Равенство $\imath\left(z_{1}, \ldots, z_{s}\right)=\left(0, z_{1}, \ldots, z_{s}\right) \in \Delta_{t}^{(s+1)}$, где $\left(z_{1}, \ldots, z_{s}\right) \in \Delta_{t}^{(s)}$, определяет естественное вложение $l: \Delta_{t}^{(s)} \rightarrow \Delta_{t}^{(s+1)}$. Индукцией по $s \geq 1$ убеждаемся, что

$$
\left|\Delta_{t}^{(s)}\right|=\sum_{i=0}^{t}\left|\Delta_{i}^{(s-1)}\right|=\left(\begin{array}{c}
s+t \\
s
\end{array}\right),\left|\Delta_{t}^{(s+1)}\right|=\left(\begin{array}{c}
s+t+1 \\
s+1
\end{array}\right) .
$$

Требование на отображения $f$ и $F$ заключаются в том, что хотя бы при одном значении $t \geq 1$ система из $\left(\begin{array}{c}s+1+t \\ s+1\end{array}\right)$ уравнений

$$
\left\{F\left(\chi_{u}^{(1)}, \ldots, \chi_{u}^{(r)}\right)=\eta_{u}, \quad u \in \Delta_{t}^{(s+1)},\right.
$$

при любых правых частях $\eta_{u} \in Y, u \in \Delta_{t}^{(s+1)}$, имеет не более одного решения относительно $r\left(\begin{array}{c}s+t \\ s\end{array}\right)$ неизвестных $\chi_{u}^{(j)} \in X, j=1, \ldots, r, u \in l\left(\Delta_{t}^{(s)}\right)$, которые в уравнения с $u \in t\left(\Delta_{t}^{(s)}\right)$ входят явно, а в остальные - опосредованно. В этой системе при каждом $j=1, \ldots, r$ остальные переменные $\chi_{u}^{(j)}$, $u \in \Delta_{t}^{(s+1)} \backslash \imath\left(\Delta_{t}^{(s)}\right)=(1,0, \ldots, 0)+\Delta_{t-1}^{(s+1)}=\left\{\left(z_{0}+1, z_{1}, \ldots, z_{s}\right) \mid\left(z_{0}, z_{1}, \ldots, z_{s}\right) \in \Delta_{t-1}^{(s+1)}\right\}$, выражаются через неизвестные $\chi_{u}^{(j)}, u \in \imath\left(\Delta_{t}^{(s)}\right)$, с помощью соотношений типа (2), именно,

$$
\chi_{u+e_{0}}^{(j)}=f\left(\chi_{u}^{(j)}, \chi_{u+e_{1}}^{(j)}, \ldots, \chi_{u+e_{s}}^{(j)}\right), \quad u \in \Delta_{t-1}^{(s+1)} .
$$

Здесь $e_{i} \in \mathbb{Z}^{s+1}, i=0,1, \ldots, s$, - вектор с единственной ненулевой координатой, равной 1 , на $i$-м месте.

Равенства (6) вначале применяются для $u \in \imath\left(\Delta_{t-1}^{(s)}\right)$. В результате через неизвестные $\chi_{u}^{(j)}, u \in t\left(\Delta_{t}^{(s)}\right)$, будут выражены все $\chi_{u}^{(j)}$ с $u \in e_{0}+\imath\left(\Delta_{t-1}^{(s)}\right)$. После этого равенства (6) применяются для $u \in e_{0}+t\left(\Delta_{t-2}^{(s)}\right)$, что дает выражения через неизвестные $\chi_{u}^{(j)}, \quad u \in l\left(\Delta_{t}^{(s)}\right)$, переменных $\chi_{u}^{(j)}$ с $u \in 2 e_{0}+\imath\left(\Delta_{t-2}^{(s)}\right)$. В результате продолжения этих вычислений переменные $\chi_{u}^{(j)}$ для $u \in \Delta_{t}^{(s+1)} \backslash \imath\left(\Delta_{t}^{(s)}\right)=\bigcup_{p=1}^{t}\left(p e_{0}+\imath\left(\Delta_{t-p}^{(s)}\right)\right) \quad$ будут $\quad$ выражены через неизвестные $\chi_{u}^{(j)}, u \in t\left(\Delta_{t}^{(s)}\right)$. 
Значение $t$ в системе (5) выбирается таким, что

$$
\log _{|X|}|Y| \cdot\left(\begin{array}{c}
s+1+t \\
s+1
\end{array}\right) \geq C \cdot r\left(\begin{array}{c}
s+t \\
s
\end{array}\right),
$$

где $C \geq 1$ - заранее задаваемая константа.

Неравенство (7), равносильное неравенству $\log _{|X|}|Y| \cdot\left(1+\frac{t}{s+1}\right) \geq C \cdot r$, является результатом логарифмирования по основанию $|X|$ неравенства $|Y|^{\left(\begin{array}{c}s+1+t \\ s+1\end{array}\right)} \geq\left(|X|^{r}\left(\begin{array}{c}\left(\begin{array}{c}s \\ s\end{array}\right) \\ )\end{array}\right)^{C}\right.$, в котором слева стоит число возможных значений набора правых частей, а справа - число возможных значений набора неизвестных, возведенное в степень $C$.

Неравенство (7) означает (так его можно трактовать), что число уравнений в системе (5) как бы в $C$ раз больше числа неизвестных. Правда, когда неизвестные и правые части системы берутся из различных алфавитов (у нас $X$ и $Y$ ), то о каком-либо сравнении числа уравнений и числа неизвестных говорить не приходится. Если же для некоторого алфавита $Z$ имеем $X=Z^{p}, Y=Z^{q}, p, q \in \mathbb{N}$, то при переходе к алфавиту $Z$ число неизвестных увеличится в $p$ раз, а число уравнений - в $q$ раз, и тогда уже можно говорить о соотношении между числом уравнений и числом неизвестных. Можно и не прибегать к алфавиту $Z$, достаточно для числа уравнений ввести поправочный множитель $\frac{q}{p}=\log _{|X|}|Y|$. В общем случае по определению считаем, что в одном уравнении в (5) содержится $\log _{|X|}|Y|$ так называемых условных уравнений. Если $Y=X^{q}$, то одно уравнение в (5) содержит $q$ условных уравнений. Неравенство (7) говорит о том, что число условных уравнений в (5) в $C$ раз больше числа неизвестных. Чем больше константа $C$ в неравенстве (7), тем правдоподобней предположение о том, что почти для всех пар отображений $f$ и $F$ система (5) имеет не более одного решения при любых правых частях.

Определение 1. Отображения $f$ и $F$ назовем условно независимыми уровня $C \geq 1$, если при выполнении неравенства (7) система уравнений (5) при любых правых частях имеет не более одного решения.

Чем больше константа $C$ в определении 1 , тем слабее требование условной независимости, накладываемое на отображения $f$ и $F$.

Общий недостаток рассматриваемых (как и многих других) генераторов псевдослучайных последовательностей состоит в том, что все знаки 
последовательности $\left\{y_{i}, i \in \mathbb{Z}\right\}$ выражаются через конечное число знаков $x_{0}, x_{1}, \ldots, x_{m-1}$. Как правило, значение $m$ существенно меньше числа реально используемых знаков $\left\{y_{i}, i \in I \subset \mathbb{Z}\right\}: m \ll|I|$. Поэтому знаки $y_{i}, i \in I$, зависимы.

При конструировании конкретных генераторов одна из задач состоит в том, чтобы сделать эту зависимость по возможности менее явной, в частности, затруднить ее использование для определения исходных знаков $x_{0}, x_{1}, \ldots, x_{m-1}$. Почти все известные в настоящее время методы восстановления $x_{0}, x_{1}, \ldots, x_{m-1}$ по заданным знакам $\left\{y_{i}, i \in I \subset \mathbb{Z}\right\}$ при $|I|=O(m)$ имеют экспоненциальную по $m \rightarrow \infty$ оценку трудоемкости.

Приводимая ниже теорема говорит о том, что асимптотически при $m \rightarrow \infty$ и ограниченных $r$ и $s$ представленные выше в самом общем виде фильтрующие генераторы не обеспечивают столь большую сложность задачи восстановления начальных знаков.

Теорема 1. Пусть отображения $f$ и $F$ являются условно независимымми уровня $C \geq 1$, пусть $\tau=\left[\left(\frac{C r}{\log _{|X|}|Y|}-1\right)(s+1)\right]$. Тогда по $(\tau+1) m$ подряд идущиим известным знакам выходной последовательности $\left\{y_{i}, i \in \mathbb{Z}\right\}$ можно восстановить $m$ подряд идущих знаков исходной рекуррентной последовательности $\quad\left\{x_{i}, i \in \mathbb{Z}\right\} \quad$ c трудоемкостью не выше $\left(r+1+\frac{s+1}{\tau}\right) \cdot\left(\begin{array}{c}s+\tau \\ s+1\end{array}\right) \cdot|X|^{r\left(\begin{array}{c}s+\tau \\ s\end{array}\right)} \cdot m$.

За единицу трудоемкости в теореме принята трудоемкость однократного вычисления значений отображений $f$ и $F$. В доказательстве конструктивно описан способ восстановления $m$ подряд идущих знаков последовательности $\left\{x_{i}, i \in \mathbb{Z}\right\}$, однако из-за столь внушительного коэффициента при $m$ в оценке трудоемкости он представляет только теоретический интерес.

Доказательство теоремы 1. Пусть имеется последовательность $\left\{y_{i}, i \in \mathbb{Z}\right\}$, полученная с помощью равенств (1) из последовательности $\left\{x_{i}, i \in \mathbb{Z}\right\}$, удовлетворяющей рекуррентному соотношению (2). Покажем, как по известным знакам $y_{1}, \ldots, y_{(\tau+1) m}$ можно однозначно восстанавливать $x_{v+l_{1}}, v=1, \ldots, m$.

При каждом $v=1, \ldots, m$ будем рассматривать значения $\chi_{u}^{(j)}=x_{v+l_{j}+\varphi(u)}$, $j=1, \ldots, r, u \in \Delta_{\tau}^{(s+1)}$, где $\varphi: \mathbb{Z}^{s+1} \rightarrow \mathbb{Z}, \varphi\left(z_{0}, z_{1}, \ldots, z_{s}\right)=z_{0} m+z_{1} k_{1}+\ldots+z_{s} k_{s}$. 
При таком задании отображения $\varphi$ из соотношений (2) следуют соотношения (6), а для правых частей в (5) имеем: $\eta_{u}=y_{v+\varphi(u)}, u \in \Delta_{\tau}^{(s+1)}$.

По очереди для каждого $v=1, \ldots, m$ будем рассматривать множество $D_{v}=\bigcup_{j=1}^{r}\left(v+l_{j}+\varphi\left(\imath\left(\Delta_{\tau}^{(s)}\right)\right)\right),\left|D_{v}\right| \leq r\left|\Delta_{\tau}^{(s)}\right|=r\left(\begin{array}{c}s+\tau \\ s\end{array}\right)$. При каждом $v$ перебираем все наборы $\left(\tilde{x}_{p}, p \in D_{v}\right) \in X^{\left|D_{v}\right|}$. Для каждых конкретных значений компонент $\tilde{x}_{p} \in X, p \in D_{v}$ применением рекуррентного соотношения (2) вычисляем значения $\tilde{x}_{q}, q \in \bigcup_{j=1}^{r}\left(v+l_{j}+\varphi\left(\Delta_{\tau}^{(s+1)}\right)\right)$. После этого по формуле (1) вычисляем значения $\tilde{y}_{p}, p \in v+\varphi\left(\Delta_{\tau}^{(s+1)}\right)$, и сравниваем их с истинными значениями $y_{p}$.

Параметр $\tau$ в формулировке теоремы выбран так, чтобы при $t=\tau$ выполнялось неравенство (7), которое равносильно неравенству $t \geq\left(\frac{C r}{\log _{|X|}|Y|}-1\right)(s+1)$. Коль скоро отображения $f$ и $F$ условно независимы уровня $C$, то только для одного вектора $\left(\tilde{x}_{p}, p \in D_{v}\right) \in X^{\left|D_{v}\right|}$ (для истинного) произойдет совпадение $\tilde{y}_{q}$ с $y_{q}$ для всех $q \in v+\varphi\left(\Delta_{\tau}^{(s+1)}\right)$. В результате будет однозначно определено значение $x_{v+l_{1}}$.

Поясним это. Полагая $\chi_{u}^{(j)}=\tilde{x}_{v+l_{j}+\varphi(u)}, j=1, \ldots, r, u \in \Delta_{\tau}^{(s+1)}$, получаем решение системы (5) с правыми частями $\eta_{u}=\tilde{y}_{v+\varphi(u)}=y_{v+\varphi(u)}, u \in \Delta_{\tau}^{(s+1)}$. Решение же может быть только одно в силу условной независимости отображений $f$ и $F$ уровня $C$.

При каждом $v=1, \ldots, m$ для отбраковки $\tilde{x}_{v+l_{1}}$ используются $y_{p}$, $v \leq p \leq v+\tau m$, поэтому для всех $v=1, \ldots, m$ потребуются только $y_{q}$, $1 \leq q \leq(\tau+1) m$.

Указанная в формулировке теоремы оценка трудоемкости следует из того, что соотношение (2) при каждом $v=1, \ldots, m$ применяется не более $r\left(\begin{array}{c}s+\tau \\ s+1\end{array}\right)|X|^{r\left(\begin{array}{l}(s+\tau) \\ s\end{array}\right)}$ раз, а соотношение $(1)-$ не более $\left(\begin{array}{c}s+\tau+1 \\ s+1\end{array}\right)|X|^{r\left(\begin{array}{l}(s+\tau) \\ s\end{array}\right)}$ раз.

Теорема 1 доказана.

За счет увеличения параметра $\tau$ требования на отображения $f$ и $F$ в формулировке теоремы 1 можно ослабить, считая их условно независи- 
мыми уровня $(C, \alpha), C \geq 1,0 \leq \alpha<1 / s$, в том смысле, что если для числа «уравнений» $N=\log _{|X|}|Y| \cdot\left(\begin{array}{c}s+1+t \\ s+1\end{array}\right)$ в системе (5) с $n=r\left(\begin{array}{c}s+t \\ s\end{array}\right)$ неизвестными в этой системе справедливо неравенство $N \geq C n^{1+\alpha}$, то имеем не более одного решения. Тогда вместо (7) нужно использовать следующее неравенство:

$$
\log _{|X|}|Y| \cdot\left(\begin{array}{c}
s+1+t \\
s+1
\end{array}\right) \geq C \cdot\left[r\left(\begin{array}{c}
s+t \\
s
\end{array}\right)\right]^{1+\alpha} .
$$

Определение 2. Отображения $f$ и $F$ назовем условно независимыми уровня $(C, \alpha), C \geq 1,0 \leq \alpha<1 / s$, если при выполнении неравенства (8) система уравнений (5) при любых правых частях имеет не более одного решения.

Чем больше значения $C, \alpha$ в определении 2, тем слабее требование условной независимости, накладываемое на отображения $f$ и $F$.

Теорема 2. Пусть отображения $f$ и $F$ являются условно независимыми уровня $(C, \alpha), \quad C \geq 1, \quad 0 \leq \alpha<1 / s, \quad$ nусть $\tau=\max \left(s+1,\left[\left(\left(\frac{C r^{1+\alpha}}{\log _{|X|}|Y|} \cdot\left(\frac{2^{s}(s+1)^{s}}{s !}\right)^{\alpha}\right)^{1 /(1-\alpha s)}-1\right)(s+1)\right]\right)$. Tогда no $(\tau+1) m$ подряд идущиим известным знакам последовательности $\left\{y_{i}, i \in \mathbb{Z}\right\}$ знаки $x_{0}, x_{1}, \ldots, x_{m-1}$ могут быть восстановлены с трудоемкостью не выше $\left(r+1+\frac{s+1}{\tau}\right) \cdot\left(\begin{array}{c}s+\tau \\ s+1\end{array}\right) \cdot|X|^{r\left(\begin{array}{c}s+\tau \\ s\end{array}\right)} \cdot m$.

Доказательство. Пусть $t>s$. Неравенство (8) переписывается в виде $\left(1+\frac{t}{s+1}\right) \log _{|X|}|Y| \geq C r^{1+\alpha}\left(\begin{array}{c}s+t \\ s\end{array}\right)^{\alpha}$. Так как $t>s$, то

$$
\begin{aligned}
\left(\begin{array}{c}
s+t \\
s
\end{array}\right) & =\prod_{k=1}^{s}\left(1+\frac{t}{k}\right)=\left(1+\frac{t}{s+1}\right)^{s} \prod_{k=1}^{s} \frac{1+\frac{t}{k}}{1+\frac{t}{s+1}}=\left(1+\frac{t}{s+1}\right)^{s} \prod_{k=1}^{s} \frac{\frac{1}{t}+\frac{1}{k}}{\frac{1}{t}+\frac{1}{s+1}} \leq \\
& \leq\left(1+\frac{t}{s+1}\right)^{s}(s+1)^{s} \prod_{k=1}^{s}\left(\frac{1}{k}+\frac{1}{t}\right) \leq\left(1+\frac{t}{s+1}\right)^{s}(s+1)^{s} \frac{2^{s}}{s !}
\end{aligned}
$$


Поэтому (8) выполняется, если $\left(1+\frac{t}{s+1}\right)^{1-\alpha s} \log _{|X|}|Y| \geq C r^{1+\alpha}\left(\frac{2^{s}(s+1)^{s}}{s !}\right)^{\alpha}$. Для доказательства теоремы 2 остается дословно повторить рассуждения из доказательства теоремы 1.

Дальнейшему ослаблению требования на отображения $f$ и $F$ в теореме 2 служит следующее определение.

Определение 3. Отображения $f$ и $F$ назовем условно независимыми, если найдется такое $\tau \in \mathbb{N}$, что для $t=\tau$ система (5) при любых правых частях имеет не более одного решения.

Замечание 1. Если отображения $f$ и $F$ являются условно независимыми, то, как следует из доказательства теоремы 1 , по $(\tau+1) m$ подряд идущим известным знакам последовательности $\left\{y_{i}, i \in \mathbb{Z}\right\}$ знаки $x_{0}, x_{1}, \ldots, x_{m-1}$ могут быть восстановлены с трудоемкостью не выше $\left(r+1+\frac{s+1}{\tau}\right) \cdot\left(\begin{array}{c}s+\tau \\ s+1\end{array}\right) \cdot|X|^{r\left(\begin{array}{l}(s+\tau \\ s\end{array}\right)} \cdot m$. В данной формулировке аналога теорем 1 и 2 нет каких-либо ориентиров на величину $\tau$, хоть она и определяется однозначно по значениям $r, s$ и отображениям $f, F$.

Автор признателен А. М. Зубкову за новое доказательство теоремы 2, которое привело к принципиальному изменению ее формулировки.

\section{Список литературы}

1. Шнайер Б. Прикладная криптография. Протоколы, алгоритмы, исходные тексты на языке СИ. - М.: ТРИУМФ, 2003.

2. Малышев $Ф$. М. Порождающие наборы элементов рекуррентных последовательностей // Труды по дискретной математике. - 2008. - Т. 11. № 2. - С. 86-111. 